\title{
Regulation of aging and cancer by enhanced environmental activation of a hypothalamic-sympathoneural-adipocyte axis
}

\author{
Quais N. Hassan II ${ }^{1,2,3 \#}$, Nicholas J. Queen ${ }^{1,2 \#}$, Lei Cao ${ }^{1,2}$ \\ ${ }^{1}$ Department of Cancer Biology and Genetics, College of Medicine, The Ohio State University, Columbus, OH, USA; ${ }^{2}$ The Ohio State University \\ Comprehensive Cancer Center, Columbus, OH, USA; ${ }^{3}$ Medical Scientist Training Program, College of Medicine, The Ohio State University, \\ Columbus, OH, USA \\ Contributions: (I) Conception and design: All authors; (II) Administrative support: L Cao; (III) Provision of study materials or patients: L Cao; (IV) \\ Collection and assembly of data: All authors; (V) Data analysis and interpretation: All authors; (VI) Manuscript writing: All authors; (VII) Final \\ approval of manuscript: All authors. \\ "These authors contributed equally to this work. \\ Correspondence to: Lei Cao, PhD. Department of Cancer Biology and Genetics, College of Medicine, The Ohio State University, 460 W 12 AVE, \\ Biomedical Research Tower Room 910, Columbus, OH 43210, USA. Email: Lei.Cao@osumc.edu.
}

\begin{abstract}
Social and environmental factors impact cancer and energy balance profoundly. Years ago, our lab established the existence of a novel brain-fat interaction we termed the "hypothalamic-sympathoneuraladipocyte (HSA) axis", through which complex environmental stimuli provided by an enriched environment regulate body composition, energy balance, and development of cancer. We have spent a significant portion of the past decade to further characterize the broad health benefits of an enriched environment (for example, leanness, enhanced immune function, and cancer resistance), and to identify mediators in the brain and periphery along the HSA axis. This review summarizes our recent work regarding the interface between endocrinology, immunology, cancer biology, aging, and neuroscience. We will discuss the interplay between these systemic phenomena and how the HSA axis can be targeted for regulation of cancer and aging.
\end{abstract}

Keywords: Aging; brain-derived neurotrophic factor (BDNF); cancer; environmental enrichment (EE); hypothalamic-sympathoneural-adipocyte axis (HSA axis)

Submitted Jan 15, 2020. Accepted for publication Feb 11, 2020.

doi: $10.21037 /$ tcr.2020.02.39

View this article at: http://dx.doi.org/10.21037/tcr.2020.02.39

\section{The HSA axis mediates the anticancer effects of environmental enrichment (EE)}

Our lab has had a long-standing interest in understanding how an individual's interaction with the social and physical environments influences health and disease. The primary model we utilize for this line of research is EE, a complex, stimulating housing environment-characterized by the use of larger-than-standard cages, abundant bedding, running wheels, mazes, toys, shelters, and increased number of social partners. Numerous studies have demonstrated that EE improves brain function and resiliency (1). These findings promoted us to ask whether an EE, known to optimize brain health, could also benefit overall health and affect the development and progression of cancer. Although the past decades have seen growing appreciation of the profound effects of environmental and lifestyle factors in shaping the initiation, promotion, and progression of cancer as well as responsiveness to treatment (2-5), the underlying mechanisms are poorly defined. Thus, we employed EE as a powerful model to elucidate mechanisms of an individual's interaction with its physical and social environment.

We assessed the effects of EE using both cancer cell implantation models and spontaneous cancer models. In our first experiment, we exposed mice to 6 weeks of EE and then subcutaneously implanted syngeneic B16 melanoma cells. We observed a remarkable suppression of melanoma growth in mice living in EE with an approximately $80 \%$ 
reduction of tumor mass compared to mice living in a standard environment (SE). Moreover, 17\% of mice living in $\mathrm{EE}$ showed no visible tumor, despite the development of palpable tumors in every SE mouse. Histopathological and signal transduction analyses found decreased cell proliferation, increased apoptosis, as well as suppression of PI3K-AKT, MAPK, and angiogenic signaling pathway activity in tumors from EE mice, consistent with slower tumor growth (6).

Next, we explored if EE was effective in other cancer types and to what extent it could be effective if implemented after established tumor growth. We implanted MC38 colon cancer cells into mice and waited until after palpable tumors occurred to initiate EE. In this pre-existing tumor model, EE resulted in over $40 \%$ reduction of tumor mass, suggesting therapeutic potential. We saw similar efficacy in an $\mathrm{APC}^{\mathrm{min} /+}$ spontaneous genetic murine model of colon cancer; EE not only decreased the total number of polyps in the small intestine of these mice by approximately $50 \%$, but also substantially reduced the size of polyps (6).

With comprehensive mechanistic studies, we identified one key pathway underlying the EE-induced anticancer phenotype, and named this brain-adipocyte communication the "hypothalamic-sympathoneural-adipocyte" (HSA) axis. The HSA axis is a specific neuroendocrine pathway linking the hypothalamus, the brain region largely responsible for coordinating and integrating the neuroendocrine and autonomic systems (7), to white adipose tissue (WAT) (8). We also demonstrated that the hypothalamic component is predominantly mediated via brain-derived neurotrophic factor (BDNF), which is upregulated by the physical, social, and cognitive stimuli provided by EE. Hypothalamic BDNF triggers the sympathetic nervous system (SNS) innervating WAT to release norepinephrine into WAT, resulting in decreased leptin expression and secretion while increased adiponectin level. The marked drop of circulating leptin, a major adipokine with pleiotropic functions, is causally associated with an anticancer phenotype (9). Since the first report of an anticancer effect of EE, others have also demonstrated that $\mathrm{EE}$ is effective in pancreatic cancer $(10,11)$, glioma (12), and lung cancer (10) models, with similar and additional mechanisms beyond the HSA axis.

\section{The HSA axis mediates the anti-obesity effects of EE}

An organism's adipose organ consists of two types of adipose tissues, WAT and brown adipose tissue (BAT). Although both are involved in lipid metabolism, WAT and BAT are distinctive at morphological, molecular, and functional levels. BAT is rich in mitochondria and metabolically active, primarily dissipating energy as heat for thermoregulation, whereas WAT is primarily responsible for storing and distributing excess energy as well as functioning as an endocrine organ secreting adipokines and other hormones. While traditionally considered as separate, accumulating evidence supports the concept of adipose plasticity. This line of thinking posits that adipocytes in each population can transform into one another to achieve a required physiological balance of heat production via BAT and energy storage via WAT [reviewed by Frontini and Cinti (13)]. Modulation of adipose plasticity has been intensively investigated in search of approaches to boost BAT for obesity prevention and treatment. BAT dissipates energy directly as heat through uncoupling fatty acid oxidation from ATP production by uncoupling protein-1 (UCP1) and is vital for the maintenance of body temperature. BAT is also involved in the regulation of body weight $(14,15)$; despite BAT composing only a small amount of total body weight ( $40-50 \mathrm{~g}$ or about $0.05-1 \%$ of body weight in adult humans in contrast to $5-10 \%$ in mouse), when fully stimulated, it is estimated that BAT can account for up to $20 \%$ of total energy expenditure, which is highly relevant with regards to control of weight (16). Moreover, even small but consistent imbalances in the energy input/ output equation can ultimately lead to obesity or leanness. Indeed, several studies demonstrate an inverse correlation between BAT activity in adult humans and body fat (17-22). Human white adipocytes from subcutaneous fat tissues can be manipulated in vitro to develop "brown" characteristics by forced expression of transcription coactivator PGC$1 \alpha(23,24)$. These findings further suggest the therapeutic potential of BAT-oriented strategies to boost energy expenditure by facilitating brown adipocyte maintenance, stimulating pre-existing brown precursors, and inducing white-to-brown transformation $(13,25,26)$. However, as Cannon and Nedergaard note, UCP1 in intact cells is constantly inhibited by purine nucleotides in the cytosol. Therefore, the strategies solely based on increasing the total amount of BAT or UCP1, may be flawed because the UCP1 is not automatically active and its inhibition has to be overcome through a process initiated physiologically by SNS stimulation (27).

Our studies on EE have revealed a new type of thermogenesis that contributes to the profound impact on body composition and metabolism in mice living in 
EE. EE induces a molecular and functional switch from WAT to BAT in the absence of chronic cold exposure or prolonged pharmacological $\beta$-adrenergic stimulation (28). The molecular characteristics of EE-induced WAT "browning", in other words, inducing brown-like cells in WAT (also called beige cells), include the induction of BAT specific markers, suppression of WAT markers, and no changes in adipocyte markers shared by both white and brown adipocytes. A 4-week exposure to EE led to a 27-fold induction of UCP1, which is more effective than a 9-fold induction observed in the same strain of mice acclimated to $5{ }^{\circ} \mathrm{C}$ cold for 4 weeks or a 23 -fold induction after injection of $\beta$-agonist CL316243 for 11-12 days (29). Of interest, EE significantly increased both $\beta 2$ - and $\beta 3$-adrenergic receptor $(\mathrm{AR})$ gene expression and protein levels in retroperitoneal WAT suggesting the absence of desensitization of $\beta$-ARs after extended exposure to $\beta$ agonist (30). After 12-week EE, obvious macroscopic changes of fat became visible to the eye with WAT turning brown and BAT visibly even darker. Our mechanistic studies demonstrate that the activation of the HSA axis mediates the EE-induced WAT browning, reduction of adiposity via increase in energy expenditure, and resistance to diet-induced obesity (DIO) (28).

With continued efforts to further characterize the HSA axis, we have also identified adipose vascular endothelial growth factor (VEGF) as a key component of the HSA axis underlying the browning effect of EE (31).VEGF is the only bona fide endothelial cell growth factor, and its presence is essential for initiation of the angiogenic program $(32,33)$. EE stimulated VEGF expression in a fat depot-specific manner and this upregulation occurred prior to the emergence of beige cells. While VEGF upregulation was independent of hypoxia, it required intact sympathetic tone to the adipose tissue. As expected, targeted adipose overexpression of VEGF by a novel engineered serotype recombinant adeno-associated virus (rAAV) vector reproduced the angiogenic and browning effect of EE. Additionally, adipose-specific VEGF knockout or pharmacological VEGF blockade by antibody abolished the beige cell recruitment induced by EE. One of the key findings of this study is that VEGF integrates multiple upstream stimulations to a common pathway that is essential to the emergence of beige cells. In addition to EE, VEGF blockade could substantially block the browning induced by the $\beta 3$-adrenergic agonist CL-316,243, the PPAR $\gamma$ ligand rosiglitazone, and voluntary running. Thus, VEGF signaling is likely a downstream pathway shared by diverse upstream mechanisms that all lead to the induction of beige cells. Targeting this common pathway may have therapeutic potential.

\section{Targeting the HSA axis for treatment of obesity and cancer}

Many cancers interact with the endocrine system. Metabolic dysregulation, especially through obesity, is not only a risk factor, but it also predicts worse therapeutic outcomes $(34,35)$. Obesity is associated with higher leptin and lower adiponectin, and the increased leptin/adiponectin ratio is thought to contribute to increased cancer risk associated with obesity. We previously discussed how EE decreases circulating leptin while increases circulating adiponectin through activating the HSA axis in mice of normal body weight. Since leptin dysregulation is known to be important in several cancers $(34,36)$, we further investigated if leptin modulation is a causative mechanism of the $\mathrm{EE}$ anti-cancer phenotype in an obese state (6). Although EE reduced body weight in obese leptin-deficient $\mathrm{Ob} / \mathrm{Ob}$ mice, it failed to inhibit B16 melanoma growth compared to $\mathrm{Ob} / \mathrm{Ob}$ mice in SE. In contrast, DIO mice with similar levels of obesity experienced both weight loss as well as reduction in tumor burden with EE $v s$. SE. Leptin supplementation of melanoma in vitro or through implantation of osmotic minipumps in $\mathrm{Ob} / \mathrm{Ob}$ mice with melanoma also resulted in hastened cancer growth (6). Together, these results implicate leptin as a key player in the $\mathrm{EE}$ anti-cancer phenotype. To assess whether leptin signaling can be targeted as a cancer therapeutic, we tested a leptin receptor neutralizing nanobody in the B16 melanoma model, and observed anti-tumor activity when injected adjacent to the tumor (37).

Because EE exerts an anticancer effect in lean state and an anti-obesity effect in obesogenic conditions, we proposed to manipulate the HSA axis for treatment of both obesity and cancer. In one study, we studied the EE's effects on spontaneous and transplanted MMTV-PyMT mammary tumorigenesis in mouse models with different adiposity and leptin signaling. DIO accelerated PyMT mammary tumor progression in SE whereas EE effectively alleviated DIO and significantly delayed the occurrence of palpable tumor in spontaneous MMTV-PyMT mice. EE also inhibited transplanted PyMT primary tumor progression in wild type DIO mice, but not in the leptin-defective $O b / O b$ mice, again confirming the role of leptin (38).

In another study, we injected AAV-BDNF to the hypothalamus to genetically activate the HSA axis. $B D N F$ 
gene therapy in middle-age DIO mice effectively decreased body weight and adiposity, improved glucose tolerance, and significantly suppressed orthotopic implanted estrogen receptor positive EO771 mammary tumor progression and completely prevented metastasis. Histological analyses of the mammary tumor found reduced angiogenesis, decreased proliferation, increased apoptosis, and reduced adipocyte recruitment and lipid accumulation in tumors from BDNFtreated mice. Interestingly, $B D N F$ gene therapy significantly reduced DIO-associated inflammation markers in the hypothalamus, adipose tissues, and serum (39). We are currently investigating whether the HSA axis affects the chronic low grade inflammation associated with obesity that is linked to increased cancer risk. Collectively, our series of studies support the notion that environmental or gentic activation of the HSA axis can ameliorate obesity in genetic $(6,38,40)$ and diet-induced $(6,28,38,39,41)$ mouse models, and inhibit cancer growth and metastasis.

\section{Immune regulation by EE via hypothalamic BDNF}

While we have established a direct link between several cancers and endocrine modulation by HSA axis activation, we have also been interested in addressing what other mechanisms initiated by EE might contribute to an anticancer phenotype (Figure 1). We briefly demonstrated that EE enhanced cytotoxicity of natural killer (NK) cells and CD8+ cytotoxic T-cells (CTL) in vitro as well as increased mobilization of these cells from the spleen in our initial B16 melanoma model (6). Recently, we have further investigated in depth how EE regulates T-cell development and function. In a model of multiple sclerosis, experimental autoimmune encephalitis (EAE), EE alleviates symptoms, inhibits spinal cord inflammation through regulation of Th1 cells and prevents EAE-induced thymic involution and disturbance. Our mechanistic studies demonstrate that hypothalamic BDNF mediates the EE's effect on the thymus through the hypothalamic-pituitary-adrenal (HPA) axis (42).

In another study, we investigated the role of CTLs in EE-induced anticancer phenotype. We found that tumors from mice in EE had fewer regulatory T-cells (Tregs) and their CTLs expressed less CD27, indicating the tumorinfiltrating CTLs were in a more activated state (43). Depletion of CD8+ T-cells significantly attenuated the tumor suppression induced by EE. These data suggest that EE's anticancer effect is mediated, at least in part, through modulation of T-cell immunity. In the spleen and lymph nodes, EE induced early phenotypic changes of T-cell populations, characterized by decreased ratio of CD4 T helper to CTL (CD4/CD8 ratio) (43). Hypothalamic overexpression of BDNF reproduced EE-induced T-cell phenotypes. Conversely, knockdown of hypothalamic BDNF blocked the T-cell modulations associated with EE. Hypothalamic BDNF exerted this immune regulation through activating both the SNS and HPA axis (43).

Other groups have shown that NK cells also contribute to the anti-tumor immunity of EE $(10,12,44)$. We recently observed that EE promoted NK cell recovery after their depletion in the spleen, bone marrow, and blood. Furthermore, EE enhanced NK cell maturation in the spleen. These improvements of NK cell reconstitution and maturation were again mediated by the hypothalamic $\mathrm{BDNF}$ (manuscript in revision at EJI).

Taken together, our findings support the notion that hypothalamic BDNF orchestrates the neuroendocrine and immune systems to confer health benefits of EE.

\section{Potential immune functions of adipose tissue in cancer}

Given the profound remodeling of adipose tissue in response to EE, we have started to explore how EE might affect adipose resident immune cells. The preliminary results are promising but it takes years to complete the mechanistic studies. Meanwhile, we developed tools to study the crosstalk between adipocyte and immune populations residing in adipose tissue. Our novel engineered hybrid rAAV serotype-Rec2, can transduce WAT and BAT far more efficiently than the naturally occurring serotypes (45). More recently, we developed a dualcassette vector system to prevent off-target transduction of liver (46). Intraperitoneal injection of Rec2 vector can achieve high transduction in all visceral fat depots with minimal transgene expression in liver. The first proof-ofconcept study showed a single intraperitoneal injection of Rec2 vector carrying leptin gene, at low dose, reversed obesity and metabolic syndromes of $\mathrm{Ob} / \mathrm{Ob}$ mice $(46,47)$.

Next, we explored the potential of targeting adipose tissue for cancer immunotherapy. Adipocyte interleukin 15 (IL-15) is important in stimulating NK cell development locally and systemically (48). IL-15 is a pro-inflammatory cytokine which increases NK cell proliferation, motility, activation, and cytotoxic effector molecule expression (49-51), as well as promotes the survival of CD8+ memory 


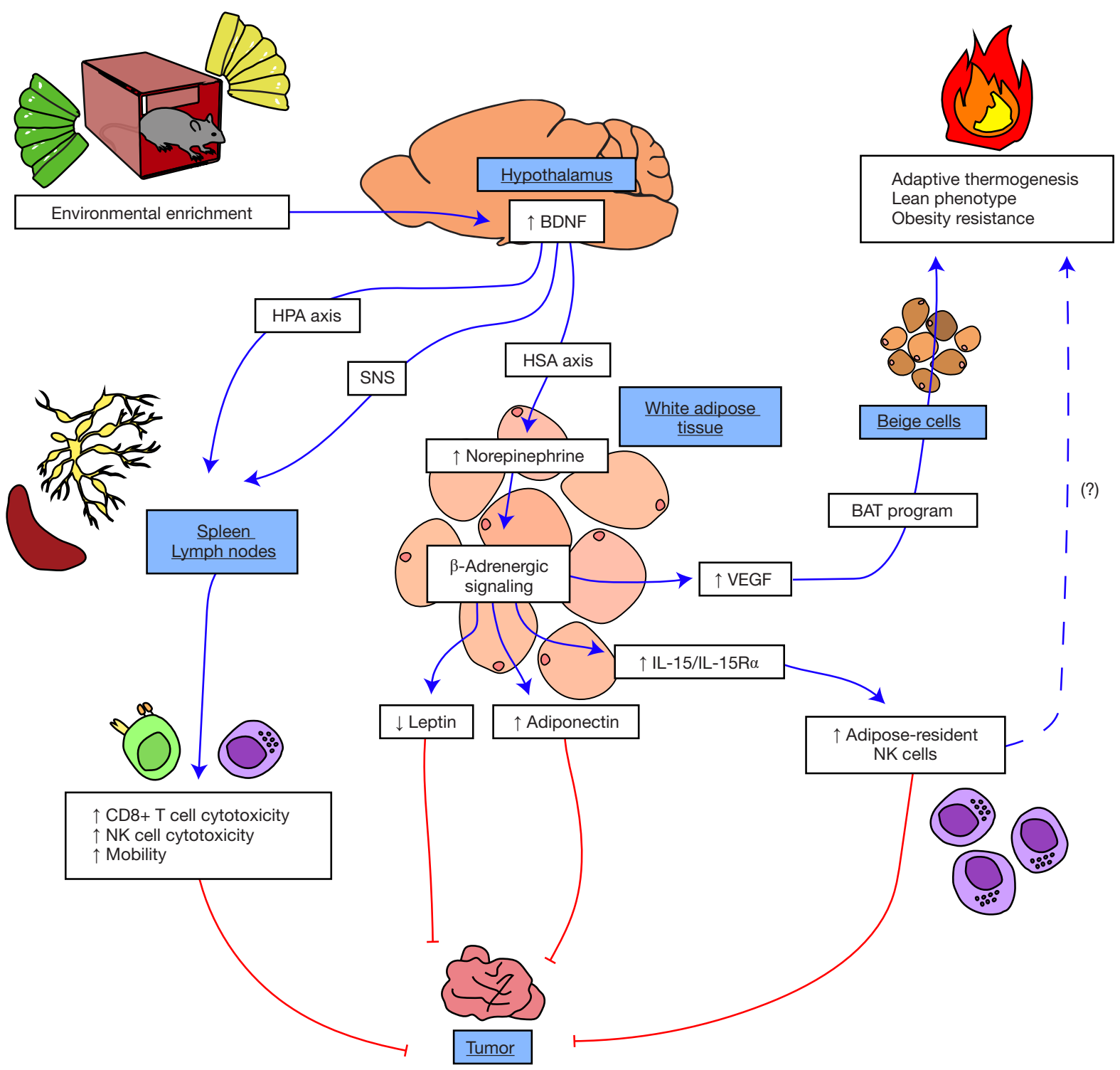

Figure 1 Mechanisms of EE-induced anticancer and anti-obesity phenotypes. EE, environmental enrichment; BDNF, brain-derived neurotrophic factor; HSA, hypothalamic-sympathoneural-adipocyte; HPA, hypothalamic-pituitary-adrenal; SNS, sympathetic nervous system; IL, interleukin; VEGF, vascular endothelial growth factor; NK, natural killer; BAT, brown adipose tissue.

T-cells and induces the proliferation, survival, and function of effector CD8+ T-cells $(52,53)$. To test this immunotherapy hypothesis, we used the adipose targeting Rec2 rAAV vector to deliver both IL-15 and its receptor, IL15-R $\alpha$ to the visceral adipose tissues by intraperitoneal injection (54). Adipose gene transfer of the IL-15/IL-15-R $\alpha$ resulted in expansion of the NK cell population in spleen and visceral fat. The adipose $I L-15 / I L-15-R \alpha$ gene therapy was effective against a subcutaneous implant of Lewis lung carcinoma (LLC) cells, and conferred a significant survival benefit in our metastatic B16-F10 melanoma model. While these results do not implicate EE or the HSA axis directly, they highlight the immune reservoir capability of adipose tissue and demonstrate that immune cell populations in adipose tissue can be manipulated to treat cancer.

\section{Mechanisms of aging}

Like cancer, aging is a complex, systemic affair. With time, all living beings undergo the aging process. This 
unavoidable nature of our biology yields numerous functional declines in most tissues, leading many to consider aging as a risk factor for disease. Here, we will briefly describe several physiological characteristics of the aging process and describe how the HSA axis and adjacent neuralimmuno-endocrine mechanisms can ameliorate or prevent age-related metabolic, immune, and behavioral deficits.

Decreases in metabolic function accompany the natural aging process. With age, animals and humans experience an increase in body mass and adiposity. Such changes are thought to result from decreased physical activity (55) and agerelated alterations in lipolysis (56), fatty acid oxidation (57), and mitochondrial function (58). Central and peripheral insulin resistance, impaired glycemic control, and sarcopenia also accompany the aging process. Age has been shown to influence circulating adipokines; most relevant to this review, increased leptin and decreased adiponectin occur with age, underlying reduced metabolic function $(59,60)$.

Additionally, age-related alterations in immune function have been observed. Centrally, microglia act as macrophagelike immune cells to survey and sense immunogenic stimuli or tissue injury. Upon insult, microglia produce proinflammatory mediators which lead to downstream induction of the immune response (61). Age-related microglial dysfunction has been associated with hyperreactivity to inflammatory stimuli, higher basal levels of cytokine expression, reduced surveilling activity, shortened process length, and slower movement (62-64). Such functional decline is associated with neurodegenerative disease and peripheral tissue dysfunction $(61,65)$. Moreover, researchers and clinicians have observed innate and adaptive immunosenescence in peripheral tissues. Such changes are characterized by reduced cytotoxicity, impairment of antigen presenting cells (APCs), reduced antibody production, reduced self-renewal capacity, and altered inflammatory responses to insult (66-68).

Aging is also associated with cognitive and behavioral changes, likely caused by reduced synapse number (69), reduced synaptic plasticity (70), reduced spine density (71), dendritic regression (71), and increased neuroinflammation. Functionally, the aging process is characterized by slowed processing speed, reduced executive function, and reduced memory and recall (72-75). Age-related structural and molecular changes within the brain can also result in altered behavior and emotionality. Reductions in brainlocalized BDNF and its associated signaling pathways have been associated with hippocampal dysfunction, memory impairment, and increased risk for schizophrenia, anxiety, and depression (76-81).

Above, we have presented a myriad of physiological change that accompanies the aging process. Fortunately, activation of the HSA axis-and other adjacent neuroimmuno-endocrine mechanisms-has been shown to ameliorate or prevent many of these age-related declines. Below, we will highlight work that describes how the HSA axis and adjacent mechanisms are implicit in healthy systemic aging.

\section{HSA axis modulation of healthy systemic aging}

Recent advances in human healthcare, food and water access, housing, and hygiene have resulted in increased global life expectancy. While improvements in human lifespan are promising, they are of lesser consequence if individuals are living longer while afflicted by acute and chronic diseases. Healthspan improvements are of upmost importance in the wake of increased global longevity; without good health, aging populations will provide a great burden for healthcare systems. Accordingly, there is a need to understand biological mechanisms through which healthy systemic aging can be increased. We have investigated whether environmental modulation of the HSA axis and adjacent neuro-immuno-endocrine mechanisms could promote healthy aging and increase healthspan in murine models. Consistent with previous work in younger mice, short-term EE (6 weeks) in middle-age mice (10 months old) was able to stimulate hypothalamic BDNF as well as associated improvements in metabolism and related molecular pathways (82). These results acted as a proof-of-concept for the use of EE in aged models, indicating that even a short exposure of EE could yield a meaningful phenotype for future studies.

Further work investigated whether the sustained exposure to complex environmental stimuli over the murine lifespan could yield increased healthspan (82). Ten-month-old mice with no prior history of enrichment were either placed in $\mathrm{SE}$ or EE for 12 months. As in previous experiments, EE mice displayed an upregulation of hypothalamic BDNF, as well as improvements in global metabolism and behavior. Mice exhibited decreased fat mass with increased lean mass, improved glycemic control, reduced hepatosteatosis, reduced hepatic glucose production, increased hepatic glucose uptake, improved motor ability, and decreased anxiety-like behavior over a year-long period by HSA axis and adjacent mechanisms. Such observations suggest that EE provides durable and significant improvements to 
healthspan in laboratory mice and can induce biological change inherent to healthy aging.

Similarly, hypothalamic administration of an rAAVBDNF vector promoted healthy aging (83) largely mimicking EE effects. BDNF gene therapy was found to prevent the development of age-related metabolic decline and reduce anxiety- and depression-like behavior. An additional cohort corroborates these metabolic findings, suggesting hypothalamic administration of rAAV-BDNF can overcome DIO in middle-aged mice (39). Together with the EE work, these data indicate the importance of the BDNF in healthy aging processes. Further work will be needed to verify whether other findings in young mice-in particular, improved immune functions-can be recapitulated in old mice.

\section{Additional brain-body implications of the HSA axis in aging}

As previously mentioned in this review, crosstalk between central and peripheral tissues is an essential tenet of our understanding of the brain-body connection. Accordingly, our lab and others have investigated functional changes in the brain which may be implicated in peripheral phenotypes. Recent work by our lab investigated whether EE could alter microglial morphology and function in aged mice (84). As in previous experiments, long-term EE was shown to improve systemic metabolism. Brain tissue immunohistochemistry revealed that $\mathrm{EE}$ increased microglial hypertrophy and ramification, with no concurrent increase in microglial cell density. Such alterations were observed alongside a reduction in neuroinflammatory markers $(C C L 2, I L 1 b$, IL6, NFKBIA, SOCS3, and H2Ab1) across several brain regions. These data suggest that $\mathrm{EE}$ can ameliorate many age-induced changes in microglial function, perhaps contributing to systemic health. Further work by our lab used pharmacological depletion of microglia in EE mice to understand the relationship between microglia and systemic/peripheral phenotypes. Microglial depletion led to improved metabolism in aged, but not young adults, supporting the hypothesis that aged microglia contribute to age-related metabolic decline. Interestingly, the EE-driven benefits on metabolism are not dependent upon microglia. Interestingly, microglial depletion did not attenuate EE effects or alter hypothalamic levels of BDNF, but rather enhanced the metabolic benefits of EE (85). Our findings demonstrate benefits from combined drug and lifestyle interventions in aged animals. Future work is needed to elucidate the underlying mechanisms.

Dietary obesity, impaired glucose tolerance, and hypertension have been linked to proinflammatory signaling within the hypothalamic regions important for feeding and energy balance $(86,87)$. Hypothalamic microinflammation — characterized by low-degree activation of proinflammatory nuclear factor kappa $\mathrm{B}(\mathrm{NF}-\kappa \mathrm{B})$ and $\mathrm{I} \kappa \mathrm{B}$ kinase $\mathrm{b}(\mathrm{IKKb})(88)$ is thought to control systemic aging processes through autocrine, paracrine, and endocrine signaling. Thus, reduced hypothalamic inflammation has been associated with improved metabolism and a reversal of aging pathologies $(86,87)$. Environmental, dietary, and pharmacological interventions to reduce hypothalamic microinflammation in models of metabolic syndrome and aging show great promise for potential therapeutics. Further work will investigate the translational aspects of these and other inflammatory mechanisms.

\section{Combining the HSA axis, cancer, and aging}

The aging process is intertwined with increased levels of DNA damage and mutational burden; such increases are thought to be due to both exogenous and endogenous factors. As such, a clear relationship between cancer and aging exists. We wish to understand how global health improvements conferred via EE may have implications for diseases like cancer or metabolic dysfunction, which worsen with age. Although the majority of our metabolism and cancer experiments have been in young or middle-age mice, it should be no surprise that we are also exploring the impact that environmental stimuli may have on these processes in aged individuals; the robustness and durability of EE phenotype in late-aged mice indicates the physiological machinery remains available throughout age, and we are optimistic about potential improvements in aged models. The association of these disease states with ageand their shared improvement in EE-suggests shared central mechanisms and encourages further exploration by us and others.

As previously described, age is thought to be a risk factor for the development of metabolic syndrome, due in part to obesity and related inflammation of adipose tissue (36). Adipose tissue inflammation is thought to contribute to systemic metabolic dysregulation via its induction of insulin, IGF-1, dyslipidemia, hyperglycemia, and oxidative stress (34). Combined with additional influences from adipokines, proinflammatory macrophages, adipose-derived stem cells, and cancerassociated adipocytes, inflamed adipose tissue creates 
a favorable microenvironment for cancer cell growth, angiogenesis, and metastasis (34). Accordingly, current work now considers the disease-implicated immune capabilities of adipose tissue (89-91), going beyond canonical metabolism and energy storage roles. In other words, age-related obesity and its related inflammation increases the likelihood that one will develop cancer. Given the growing population of aged, obese individuals, there is a pressing need to understand mechanisms inherent to the metabolic roots of cancer and to further develop preventative therapeutic tools.

Experiments previously described in this review highlight how EE and hypothalamic BDNF can improve metabolic and immune function, contributing to an anti-cancer phenotype, however, many of these experiments were performed in young or otherwise healthy mice. Disease models frequently fail to consider common and realistic systemic conditions such as age or metabolic dysfunction, perhaps out of feasibility or to minimize confounding factors. Fortunately, the robust and repeatable nature of the EE-BDNF phenotype has been exhibited across both young and old mice. Consideration of these and other realistic systemic conditions will aid in determining to what extent EE can ameliorate or prevent disease in aged models. Additional investigation of immunoendocrine players within the aged tumor microenvironment and adipose tissue will be necessary to determine the full extent of EE and BDNF mediated anti-cancer mechanisms and establish possible therapeutics for aged populations.

\section{Discussion and future directions}

As the world's population collectively experiences longer lives and better health in youth, efforts to improve treatments for diseases associated with age become increasingly worthwhile. Biomedical researchers are becoming increasingly aware of how the interconnectedness of many physiological systems complicates not only research experiments but also health. We believe that EE serves as one powerful tool to study the brain-body connection, providing valuable mechanistic insight regarding central control over peripheral and systemic processes in health and disease. Future work will attempt to elucidate tissue- and cellular-level mechanisms within a myriad of tissues, including: skeletal muscle, heart, lungs, liver, kidney, pancreas, intestines, and hematopoietic and lymphatic tissues. Crosstalk between these organs will aid in elucidation of neural-immuno-endocrine mechanisms and will continue to be a future area of focus in the fields of cancer and metabolism (Figure 1) as well as aging (Figure 2).

Incorporation of-omics level techniques will be necessary to fully appreciate the crosstalk-heavy nature of EE and HSA axis signaling. Characterization of these signals at a local level would similarly benefit from further exploration of adipose depot, nerve, hematopoietic, and tumor microenvironments. It will be interesting to investigate cell-to-cell and tissue-to-tissue relationships and reveal their novel crosstalk mechanisms in this manner while incorporating additional tools like epigenetic assays, flow cytometry and cell sorting, and mass spectrometry, which allow for the powerful separation and identification required to tease apart such complex systems. Specifically, regulation of gene expression, histological organization of tissues, identity and localization of metabolites, and interactions with the extracellular matrix are some areas we have particularly identified as worthy of investigation given advances in available acquisition and analysis technology.

Crosstalk implies multi-directionality. While much of our focus has been from brain to adipocytes, it is important to note that feedback loops and other mechanisms may play a role in neuroendocrine pathways. Indeed, our recent work with phosphatase and tensin homologue deleted on chromosome ten (PTEN) provides one such example (92). PTEN is multifunctional, acting as a tumor suppressor and a lipid phosphatase; the latter role implicates PTEN as an important regulator of cellular growth, survival, and insulin mediated glucose uptake. Using various experimental methods, we discovered a previously unexamined role of PTEN as a potent regulator of adipose tissue homeostasis and adipokine secretion. PTEN knockdown in individual adipose depot resulted in massive expansion of the affected fat depot and induced leptin surge in circulation. The leptin surge in turn acted centrally-within the hypothalamusto elevate sympathetic tone to other adipose depots and thereby increase PTEN, stimulate lipolysis, and reduce fat mass to maintain a set point of whole-body adiposity. The discovery of this "adipose PTEN-leptin-SNS" feedback loop highlights the importance of brain-adipose crosstalk in whole-body homeostasis and other systemic processes.

Our and others' work with the EE model has demonstrated how complex environmental stimuli can affect health and disease. In humans, lifestyle and environmental factors affect cancer prognosis, and in some cases are thought to contribute in a greater fashion than inherited genetic defects alone. Excess weight gain is one of the largest avoidable causes of cancer and mortality, with poor diet and lack of physical activity being primary 


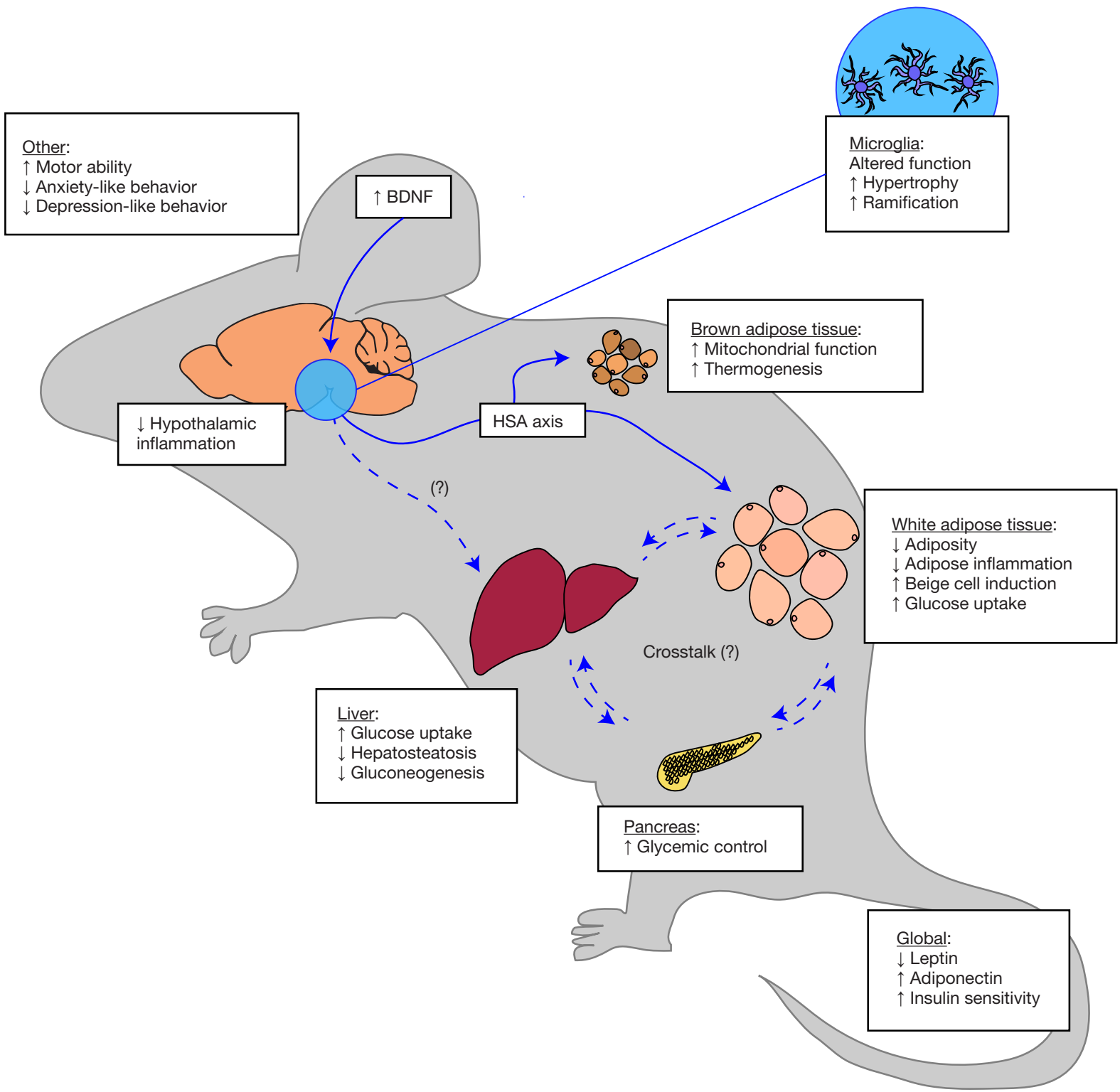

Figure 2 Mechanisms of EE promotion of healthy aging. EE, environmental enrichment; BDNF, brain-derived neurotrophic factor; HSA, hypothalamic-sympathoneural-adipocyte.

environmental contributors (34). While the search for pharmacological panaceas is ongoing, it is important to consider how environmental interventions might yield similar improvements in global health for young and aged individuals. Obvious improvements in diet and physical activity mitigate obesity, but work with EE has caused our lab to consider the importance of other, less-intuitive environmental factors and their contribution to local adipose and systemic inflammation. Mental stimulation, social partners, and additional complex stimuli may play a role in healthy aging and anti-cancer processes. As discussed previously $(6,28,82)$, the full benefits of EE could not be recapitulated with exercise alone, indicating the importance of other factors in environmental activation of the HSA axis and adjacent neural-immuno-endocrine mechanisms.

In sum, our understanding of the effects of environmental stimulation on the development of cancer have grown substantially since our 2012 review, with an emphasis on local and systemic inflammation, aging, and the immunoendocrine role of adipose tissue (8). Future 
work by our lab will seek to explore the intersections of cancer, metabolism, and aging, with special attention paid to both systemic physiological interactions as well as tissuelevel cellular and molecular mechanisms.

\section{Acknowledgments}

We express our gratitude to our lab's past and current members for enriching our lab environment; this has made our work not only possible, but truly enjoyable.

Funding: Funding for this work was provided by NIH grants CA163640, CA166590, and AG041250 to Lei Cao.

\section{Footnote}

Provenance and peer review: This article was commissioned by the Guest Editor (Nathan A. Berger) for the series "Energy Balance, Aging, Obesity, and Cancer" published in Translational Cancer Research. The article was sent for external peer review organized by the Guest Editor and editorial office.

Conflicts of Interest: All authors have completed the ICMJE uniform disclosure form (available at http://dx.doi. org/10.21037/tcr.2020.02.39). The series "Energy Balance, Aging, Obesity, and Cancer" was commissioned by the editorial office without any funding or sponsorship. Lei Cao is an inventor on US patent 9,265,843 B2 on the use of an autoregulatory BDNF vector. The authors have no other conflicts of interest to declare.

Ethical Statement: The authors are accountable for all aspects of the work in ensuring that questions related to the accuracy or integrity of any part of the work are appropriately investigated and resolved.

Open Access Statement: This is an Open Access article distributed in accordance with the Creative Commons Attribution-NonCommercial-NoDerivs 4.0 International License (CC BY-NC-ND 4.0), which permits the noncommercial replication and distribution of the article with the strict proviso that no changes or edits are made and the original work is properly cited (including links to both the formal publication through the relevant DOI and the license). See: https://creativecommons.org/ licenses/by-nc-nd/4.0/.

\section{References}

1. Nithianantharajah J, Hannan AJ. Enriched environments, experience-dependent plasticity and disorders of the nervous system. Nat Rev Neurosci 2006;7:697-709.

2. Castaño Z, Tracy K, McAllister SS. The tumor macroenvironment and systemic regulation of breast cancer progression. Int J Dev Biol 2011;55:889-97.

3. Green McDonald P, O'Connell M, Lutgendorf SK. Psychoneuroimmunology and cancer: a decade of discovery, paradigm shifts, and methodological innovations. Brain Behav Immun 2013;30 Suppl:S1-9.

4. Kolonel LN, Altshuler D, Henderson BE. The multiethnic cohort study: exploring genes, lifestyle and cancer risk. Nat Rev Cancer 2004;4:519-27.

5. Baade PD, Youlden DR, Krnjacki LJ. International epidemiology of prostate cancer: geographical distribution and secular trends. Mol Nutr Food Res 2009;53:171-84.

6. Cao L, Liu X, Lin EJ, et al. Environmental and genetic activation of a brain-adipocyte BDNF/leptin axis causes cancer remission and inhibition. Cell 2010;142:52-64.

7. Ulrich-Lai YM, Herman JP. Neural regulation of endocrine and autonomic stress responses. Nat Rev Neurosci 2009;10:397-409.

8. Cao L, During MJ. What is the brain-cancer connection? Annu Rev Neurosci 2012;35:331-45.

9. Slater AM, Cao L. A protocol for housing mice in an enriched environment. J Vis Exp 2015;(100):e52874.

10. Song Y, Gan Y, Wang Q, et al. Enriching the housing environment for mice enhances their NK cell antitumor immunity via sympathetic nerve-dependent regulation of NKG2D and CCR5. Cancer Res 2017;77:1611-22.

11. Li G, Gan Y, Fan Y, et al. Enriched environment inhibits mouse pancreatic cancer growth and down-regulates the expression of mitochondria-related genes in cancer cells. Sci Rep 2015;5:7856.

12. Garofalo S, D'Alessandro G, Chece G, et al. Enriched environment reduces glioma growth through immune and non-immune mechanisms in mice. Nat Commun 2015;6:6623.

13. Frontini A, Cinti S. Distribution and development of brown adipocytes in the murine and human adipose organ. Cell Metab 2010;11:253-6.

14. Enerbäck S, Jacobsson A, Simpson EM, et al. Mice lacking mitochondrial uncoupling protein are cold-sensitive but not obese. Nature 1997;387:90-4.

15. Feldmann HM, Golozoubova V, Cannon B, et al. UCP1 
ablation induces obesity and abolishes diet-induced thermogenesis in mice exempt from thermal stress by living at thermoneutrality. Cell Metab 2009;9:203-9.

16. Rothwell NJ, Stock MJ. Luxuskonsumption, diet-induced thermogenesis and brown fat: the case in favour. Clin Sci (Lond) 1983;64:19-23.

17. Saito M, Okamatsu-Ogura Y, Matsushita M, et al. High incidence of metabolically active brown adipose tissue in healthy adult humans: effects of cold exposure and adiposity. Diabetes 2009;58:1526-31.

18. Nedergaard J, Bengtsson T, Cannon B. Unexpected evidence for active brown adipose tissue in adult humans. Am J Physiol Endocrinol Metab 2007;293:E444-52.

19. van Marken Lichtenbelt WD, Vanhommerig JW, Smulders NM, et al. Cold-activated brown adipose tissue in healthy men. N Engl J Med 2009;360:1500-8.

20. Virtanen KA, Lidell ME, Orava J, et al. Functional brown adipose tissue in healthy adults. $\mathrm{N}$ Engl J Med 2009;360:1518-25.

21. Zingaretti MC, Crosta F, Vitali A, et al. The presence of UCP1 demonstrates that metabolically active adipose tissue in the neck of adult humans truly represents brown adipose tissue. FASEB J 2009;23:3113-20.

22. Yoneshiro T, Aita S, Matsushita M, et al. Brown adipose tissue, whole-body energy expenditure, and thermogenesis in healthy adult men. Obesity (Silver Spring) 2011;19:13-6.

23. Tiraby C, Tavernier G, Lefort C, et al. Acquirement of brown fat cell features by human white adipocytes. J Biol Chem 2003;278:33370-6.

24. Mazzucotelli A, Viguerie N, Tiraby C, et al. The transcriptional coactivator peroxisome proliferator activated receptor (PPAR)gamma coactivator-1 alpha and the nuclear receptor PPAR alpha control the expression of glycerol kinase and metabolism genes independently of PPAR gamma activation in human white adipocytes. Diabetes 2007;56:2467-75.

25. Enerbäck S. Brown adipose tissue in humans. Int J Obes (Lond) 2010;34 Suppl 1:S43-6.

26. Kajimura S, Seale P, Spiegelman BM. Transcriptional control of brown fat development. Cell Metab 2010;11:257-62.

27. Nedergaard J, Cannon B. The changed metabolic world with human brown adipose tissue: therapeutic visions. Cell Metab 2010;11:268-72.

28. Cao L, Choi EY, Liu X, et al. White to brown fat phenotypic switch induced by genetic and environmental activation of a hypothalamic-adipocyte axis. Cell Metab
2011;14:324-38.

29. Vegiopoulos A, Müller-Decker K, Strzoda D, et al. Cyclooxygenase-2 controls energy homeostasis in mice by de novo recruitment of brown adipocytes. Science 2010;328:1158-61.

30. Arner P. Adrenergic receptor function in fat cells. Am J Clin Nutr 1992;55:228S-36S.

31. During MJ, Liu X, Huang W, et al. Adipose VEGF links the white-to-brown fat switch with environmental, genetic, and pharmacological stimuli in male mice. Endocrinology 2015;156:2059-73.

32. Papetti M, Herman IM. Mechanisms of normal and tumor-derived angiogenesis. Am J Physiol Cell Physiol 2002;282:C947-70.

33. Zhang QX, Magovern CJ, Mack CA, et al. Vascular endothelial growth factor is the major angiogenic factor in omentum: mechanism of the omentum-mediated angiogenesis. J Surg Res 1997;67:147-54.

34. Deng T, Lyon CJ, Bergin S, et al. Obesity, Inflammation, and Cancer. Annu Rev Pathol 2016;11:421-49.

35. Calle EE, Rodriguez C, Walker-Thurmond K, et al. Overweight, obesity, and mortality from cancer in a prospectively studied cohort of U.S. adults. N Engl J Med 2003;348:1625-38.

36. De Pergola G, Silvestris F. Obesity as a major risk factor for cancer. J Obes 2013;2013:291546.

37. McMurphy T, Xiao R, Magee D, et al. The anti-tumor activity of a neutralizing nanobody targeting leptin receptor in a mouse model of melanoma. PLoS One 2014;9:e89895.

38. Foglesong GD, Queen NJ, Huang W, et al. Enriched environment inhibits breast cancer progression in obese models with intact leptin signaling. Endocr Relat Cancer 2019;26:483-95.

39. Liu X, McMurphy T, Xiao R, et al. Hypothalamic gene transfer of BDNF inhibits breast cancer progression and metastasis in middle age obese mice. Mol Ther 2014;22:1275-84.

40. Siu JJ, Queen NJ, Liu X, et al. Molecular Therapy of Melanocortin-4-Receptor Obesity by an Autoregulatory BDNF Vector. Mol Ther Methods Clin Dev 2017;7:83-95.

41. Cao L, Lin EJ, Cahill MC, et al. Molecular therapy of obesity and diabetes by a physiological autoregulatory approach. Nat Med 2009;15:447-54.

42. Xiao R, Bergin SM, Huang W, et al. Enriched environment regulates thymocyte development and alleviates experimental autoimmune encephalomyelitis in mice. Brain Behav Immun 2019;75:137-48. 
43. Xiao R, Bergin SM, Huang W, et al. Environmental and genetic activation of hypothalamic BDNF modulates T-cell immunity to exert an anticancer phenotype. Cancer Immunol Res 2016;4:488-97.

44. Meng Z, Liu T, Song Y, et al. Exposure to an enriched environment promotes the terminal maturation and proliferation of natural killer cells in mice. Brain Behav Immun 2019;77:150-60.

45. Liu X, Magee D, Wang C, et al. Adipose tissue insulin receptor knockdown via a new primate-derived hybrid recombinant AAV serotype. Mol Ther Methods Clin Dev 2014. doi: $10.1038 / \mathrm{mtm} .2013 .8$.

46. Huang W, Liu X, Queen NJ, et al. Targeting visceral fat by intraperitoneal delivery of novel AAV serotype vector restricting off-target transduction in liver. Mol Ther Methods Clin Dev 2017;6:68-78.

47. Huang W, Queen NJ, Cao L. rAAV-mediated gene delivery to adipose tissue. Methods Mol Biol 2019;1950:389-405.

48. Liou YH, Wang SW, Chang CL, et al. Adipocyte IL15 regulates local and systemic NK cell development. J Immunol 2014;193:1747-58.

49. Carson WE, Giri JG, Lindemann MJ, et al. Interleukin (IL) 15 is a novel cytokine that activates human natural killer cells via components of the IL-2 receptor. J Exp Med 1994;180:1395-403.

50. Vosshenrich CA, Ranson T, Samson SI, et al. Roles for common cytokine receptor gamma-chain-dependent cytokines in the generation, differentiation, and maturation of NK cell precursors and peripheral NK cells in vivo. J Immunol 2005;174:1213-21.

51. Fehniger TA, Caligiuri MA. Interleukin 15: biology and relevance to human disease. Blood 2001;97:14-32.

52. Cheng L, Du X, Wang Z, et al. Hyper-IL-15 suppresses metastatic and autochthonous liver cancer by promoting tumour-specific CD8+ T cell responses. J Hepatol 2014;61:1297-303.

53. Schluns KS, Williams K, Ma A, et al. Cutting edge: requirement for IL-15 in the generation of primary and memory antigen-specific CD8 T cells. J Immunol 2002;168:4827-31.

54. Xiao R, Mansour AG, Huang W, et al. Adipocytes: a novel target for IL-15/IL-15R $\alpha$ cancer gene therapy. Mol Ther 2019;27:922-32.

55. White Z, Terrill J, White RB, et al. Voluntary resistance wheel exercise from mid-life prevents sarcopenia and increases markers of mitochondrial function and autophagy in muscles of old male and female C57BL/6J mice. Skelet
Muscle 2016;6:45.

56. Mennes E, Dungan CM, Frendo-Cumbo S, et al. Agingassociated reductions in lipolytic and mitochondrial proteins in mouse adipose tissue are not rescued by metformin treatment. J Gerontol A Biol Sci Med Sci 2014;69:1060-8.

57. Houtkooper RH, Argmann C, Houten SM, et al. The metabolic footprint of aging in mice. Sci Rep 2011;1:134.

58. Katic M, Kennedy AR, Leykin I, et al. Mitochondrial gene expression and increased oxidative metabolism: role in increased lifespan of fat-specific insulin receptor knock-out mice. Aging Cell 2007;6:827-39.

59. Palmer AK, Kirkland JL. Aging and adipose tissue: potential interventions for diabetes and regenerative medicine. Exp Gerontol 2016;86:97-105.

60. Atzmon G, Pollin TI, Crandall J, et al. Adiponectin levels and genotype: a potential regulator of life span in humans. J Gerontol A Biol Sci Med Sci 2008;63:447-53.

61. Guillemot-Legris O, Muccioli GG. Obesity-induced neuroinflammation: beyond the hypothalamus. Trends Neurosci 2017;40:237-53.

62. Ziebell JM, Adelson PD, Lifshitz J. Microglia: dismantling and rebuilding circuits after acute neurological injury. Metab Brain Dis 2015;30:393-400.

63. Norden DM, Muccigrosso MM, Godbout JP. Microglial priming and enhanced reactivity to secondary insult in aging, and traumatic CNS injury, and neurodegenerative disease. Neuropharmacology 2015;96:29-41.

64. Hefendehl JK, Neher JJ, Sühs RB, et al. Homeostatic and injury-induced microglia behavior in the aging brain. Aging Cell 2014;13:60-9.

65. Spittau B. Aging microglia-phenotypes, functions and implications for age-related neurodegenerative diseases. Front Aging Neurosci 2017;9:194.

66. Mocchegiani E, Malavolta M. NK and NKT cell functions in immunosenescence. Aging Cell 2004;3:177-84.

67. Ito K, Hirao A, Arai F, et al. Regulation of oxidative stress by ATM is required for self-renewal of haematopoietic stem cells. Nature 2004;431:997-1002.

68. Lefebvre JS, Maue AC, Eaton SM, et al. The aged microenvironment contributes to the age-related functional defects of CD4 T cells in mice. Aging Cell 2012;11:732-40.

69. Peters A, Sethares C, Luebke JI. Synapses are lost during aging in the primate prefrontal cortex. Neuroscience 2008;152:970-81.

70. Hatanpää K, Isaacs KR, Shirao T, et al. Loss of proteins regulating synaptic plasticity in normal aging of the human 
brain and in Alzheimer disease. J Neuropathol Exp Neurol 1999;58:637-43.

71. Dickstein DL, Weaver CM, Luebke JI, et al. Dendritic spine changes associated with normal aging. Neuroscience 2013;251:21-32.

72. Verhaeghen P, Salthouse TA. Meta-analyses of agecognition relations in adulthood: estimates of linear and nonlinear age effects and structural models. Psychol Bull 1997;122:231-49.

73. Salthouse TA. Aging and measures of processing speed. Biol Psychol 2000;54:35-54.

74. Spencer WD, Raz N. Differential effects of aging on memory for content and context: a meta-analysis. Psychol Aging 1995; 10:527-39.

75. Zacks RT, Hasher L, Li KZH. Human memory. In: Salthouse TA, Craik FIM. Handbook of aging and cognition. 2nd ed. Mahwah: Lawrence Erlbaum, 2000:293-357.

76. Egan MF, Kojima M, Callicott JH, et al. The BDNF val66met polymorphism affects activity-dependent secretion of BDNF and human memory and hippocampal function. Cell 2003;112:257-69.

77. Erickson KI, Miller DL, Roecklein KA. The aging hippocampus: interactions between exercise, depression, and BDNF. Neuroscientist 2012;18:82-97.

78. Martinowich K, Manji H, Lu B. New insights into BDNF function in depression and anxiety. Nat Neurosci 2007;10:1089-93.

79. Angelucci F, Brenè S, Mathé AA. BDNF in schizophrenia, depression and corresponding animal models. Mol Psychiatry 2005;10:345-52.

80. Laske C, Stellos K, Hoffmann N, et al. Higher BDNF serum levels predict slower cognitive decline in Alzheimer's disease patients. Int J Neuropsychopharmacol 2011;14:399-404.

81. Lampinen P, Heikkinen RL, Ruoppila I. Changes in intensity of physical exercise as predictors of depressive symptoms among older adults: an eight-year follow-up.

Cite this article as: Hassan QN 2nd, Queen NJ, Cao L. Regulation of aging and cancer by enhanced environmental activation of a hypothalamic-sympathoneural-adipocyte axis. Transl Cancer Res 2020;9(9):5687-5699. doi: 10.21037/ tcr.2020.02.39
Prev Med 2000;30:371-80.

82. McMurphy T, Huang W, Queen NJ, et al. Implementation of environmental enrichment after middle age promotes healthy aging. Aging (Albany NY) 2018;10:1698-721.

83. McMurphy T, Huang W, Liu X, et al. Hypothalamic gene transfer of BDNF promotes healthy aging in mice. Aging Cell 2019;18:e12846.

84. Ali S, Liu X, Queen NJ, et al. Long-term environmental enrichment affects microglial morphology in middle age mice. Aging (Albany NY) 2019;11:2388-402.

85. Ali S, Mansour AG, Huang W, et al. CSF1R inhibitor PLX5622 and environmental enrichment additively improve metabolic outcomes in middle-aged female mice. Aging (Albany NY) 2020;12:2101-22.

86. Cai D, Khor S. "Hypothalamic microinflammation" paradigm in aging and metabolic diseases. Cell Metab 2019;30:19-35.

87. Tang Y, Purkayastha S, Cai D. Hypothalamic microinflammation: a common basis of metabolic syndrome and aging. Trends Neurosci 2015;38:36-44.

88. Zhang $\mathrm{X}$, Zhang G, Zhang $\mathrm{H}$, et al. Hypothalamic IKKbeta/NF-kappaB and ER stress link overnutrition to energy imbalance and obesity. Cell 2008;135:61-73.

89. Deng T, Lyon CJ, Minze LJ, et al. Class II major histocompatibility complex plays an essential role in obesity-induced adipose inflammation. Cell Metab 2013;17:411-22.

90. Nishimura S, Manabe I, Nagasaki M, et al. CD8+ effector $T$ cells contribute to macrophage recruitment and adipose tissue inflammation in obesity. Nat Med 2009;15:914-20.

91. Tilg H, Moschen AR. Adipocytokines: mediators linking adipose tissue, inflammation and immunity. Nat Rev Immunol 2006;6:772-83.

92. Huang W, Queen NJ, McMurphy TB, et al. Adipose PTEN regulates adult adipose tissue homeostasis and redistribution via a PTEN-leptin-sympathetic loop. Mol Metab 2019;30:48-60. 